\title{
Physikerinnen und Physiker im Beruf
}

\author{
Dr. Anja Metzelthin \\ Referentin des Vorstands für die Ressorts Schule \\ sowie Industrie, Wirtschaft und Berufsfragen \\ Deutsche Physikalische Gesellschaft e. V. \\ Dr. Oliver Koppel \\ Senior Economist für Innovationen und MINT \\ Institut der Deutschen Wirtschaft Köln
}

Seit über 40 Jahren beobachtet die Deutsche Physikalische Gesellschaft (DPG) den Arbeitsmarkt für Physikerinnen und Physiker und berichtet ihren Mitgliedern regelmäßig. Allerdings ist die Erfassung der Daten speziell für Physiker nicht trivial, da sie in den meisten Publikationen gemeinsam mit anderen Natur- oder Ingenieurwissenschaften aufgeführt werden. Daher wertet die DPG diese Daten selbst aus.

Jeder, der einmal Physik studiert hat, weiß, dass viele der ehemaligen Kommilitonen irgendwann die klassischen 
Physikberufe verlassen und erfolgreich in unzähligen anderen Branchen und Berufen arbeiten. Es gibt kaum eine andere Gruppe, die eine so hohe Arbeitsmarktflexibilität zeigt. Dies macht die Erhebung von Daten allerdings noch komplexer. Aus diesem Grund müssen wir Sie durch einige Definitionen führen.

Die Daten, auf denen dieser Abschnitt beruht, stammen im Wesentlichen aus zwei Quellen:

1. Zahlen zu Arbeitslosen- und Beschäftigtenzahlen sowie offenen Stellen von der Bundesagentur für Arbeit (BA): Die umfassende Datenbank der BA liefert Zahlen über diejenigen Personen, die sich bei der BA arbeitslos gemeldet haben und in der beruflichen Tätigkeit als Physiker in ein neues Arbeitsverhältnis vermittelt werden wollen. Welche Ausbildung diejenige Person hat, wird dabei nicht erfasst. Daher umfassen die Daten der BA lediglich die Teilgruppe, die in den für Physiker definierten Berufsgruppen arbeitet. Selbstständige, Beamte etc. sind nicht erfasst.

2. Zahlen des Mikrozensus, der repräsentativen $1 \%$-Stichprobe des Statistischen Bundesamtes über alle deutschen Haushalte: Der Mikrozensus ist die amtliche Repräsentativstatistik über die Bevölkerung und den Arbeitsmarkt und beinhaltet sowohl die Studienrichtung des formalen Bildungsabschlusses als auch die Beschäftigung, der eine Person nachgeht. Als $1 \%$-Zufallsstichprobe über alle Haushalte ist der Mikrozensus mit unvermeidbaren zufallsbedingten Stichprobenfehlern behaftet. Die in diesem Abschnitt aufgeführten Werte, die auf die Gesamtbevölkerung hochgerechnet sind, haben bei einer Zellenbesetzung von über 100.000 einen einfachen relativen Standardfehler unter $4 \%$, bei Zellenbesetzungen unter 10.000 hingegen über $10 \%$. Das Jahr 2014 repräsentiert den bei Erstellung des Artikels aktuellsten verfügbaren Datenstand. 


\section{Wer ist Physiker?}

Als Physiker werden alle Personen verstanden, die einen akademischen Abschluss in einem Studiengang mit der Hauptfachrichtung Physik erreicht haben. Wir sprechen in diesem Fall vom Ausbildungsberuf Physiker. Personen, die ein Lehramtsstudium im Fach Physik absolviert haben, können im Mikrozensus nicht berücksichtigt werden, da dieser die Abschlüsse nicht nach Fachrichtung erfasst. Einen Sonderfall stellen Absolventen des interdisziplinären Fachhochschul-Studiengangs Physikalische Technik dar, dessen Inhalte stark bis schwerpunktmäßig physikalisch geprägt sind. Da die Zuordnung der Hauptfachrichtung im Mikrozensus auf Selbstauskunft beruht, ordnen sich diese Absolventen im Mikrozensus in der Regel der Hauptfachrichtung Physik zu, wenngleich ihr Abschluss in der offiziellen Hochschulstatistik in der ingenieurwissenschaftlichen Hauptfachrichtung Maschinenbau/Verfahrenstechnik geführt wird.

Der Erwerbsberuf Physiker wird tätigkeitsbasiert und folglich völlig anders als der am formalen Bildungsabschluss ansetzende Ausbildungsberuf Physiker erfasst. Im Erwerbsberuf Physiker werden Personen erfasst, die z. B. Gesetzmäßigkeiten in der unbelebten Natur erkennen und in mathematische Modelle fassen. Ganz konkret verwendet u. a. die BA ein System der Berufsklassifizierungen, die sog. Klassifikation der Berufe (KldB 2010) [1], bei der dem Erwerbsberuf Physiker drei Kennziffern zugeordnet werden. Alle Personen in Berufen, die diesen Kennziffern zugeordnet werden, zählen zum Erwerbsberuf Physiker. Nicht dazu gehören z. B. Personen mit Abschluss eines Physikstudiums, die im Management (wirtschaftswissenschaftlicher Erwerbsberuf) oder als Professoren (den Lehr- und Forschungsberufen zugeordnet) arbeiten. Auch wenn es seltsam klingt, wird der Physikprofessor nicht dem Erwerbsberuf Physiker zugeordnet, sondern den Lehrberufen. 


\section{Demografie}

Gemäß Mikrozensus 2014 gibt es in Deutschland 149.600 Physikerinnen und Physiker im Sinne des Ausbildungsberufs, von denen 110.200 einer Erwerbstätigkeit nachgehen. Hierbei ist zu beachten, dass die Zahlen keine Studierenden beinhalten, sondern Personen mit einem getätigten Abschluss (s. oben). Deren Aufteilung nach Alterskohorten, Erwerbstätigkeit und Erwerbstätigenquoten findet sich in Tab. 1 .

Dass sich die Erwerbstätigenquoten über die Alterskohorten verändern, ist normal. In den ersten Berufsjahren sind sie etwas niedriger; häufig gibt es nach dem Studienabschluss eine temporäre Sucharbeitslosigkeit. Häufig sind auch gerade in jüngeren Jahren andere Faktoren wichtig, z. B. familiäre Auszeiten aufgrund von Kindern, sodass Personen dem Arbeitsmarkt nicht zur Verfügung stehen. Nach einem Maximum in der Alterskohorte 40-49 Jahre nimmt die Erwerbstätigenquote wieder $\mathrm{ab}$ - zunächst nur leicht, was beispielsweise damit zu erklären ist, dass vereinzelt Personen in den Vorruhestand gehen. Mit einem Wert von 93,3\% liegt die Erwerbstätigenquote 50- bis 59-jähriger Physiker jedoch nicht nur auf einem sehr hohen Niveau, sondern auch

Tab. 1 Altersstruktur und Erwerbstätigenquoten von Physikern in Deutschland [2]

\begin{tabular}{llll}
\hline Jahr: 2014 & Physiker & $\begin{array}{l}\text { Darunter: } \\
\text { Erwerbstätige }\end{array}$ & $\begin{array}{l}\text { Erwerbstätigen- } \\
\text { quoten }\end{array}$ \\
\hline (in \%)
\end{tabular}

aErwerbstätigenquote: Anteil der erwerbstätigen Physiker an allen Physikern 
deutlich höher als bei gleichaltrigen sonstigen Akademikern (90,2\%). Ob dies daran liegt, dass Physiker zu einem höheren Anteil als andere Berufsgruppen in fortgeschrittenem Alter arbeiten oder dass der Anteil an Männern in der Physik höher ist, kann aufgrund der zu geringen Fallzahlen nicht ermittelt werden.

Bei der Interpretation der Erwerbstätigenquote im letzten Alterssegment muss berücksichtigt werden, dass hier im Nenner sämtliche Physiker im Alter ab 60 Jahren berücksichtigt werden, von denen jedoch mit zunehmendem Alter immer weniger tatsächlich noch dem Arbeitsmarkt zur Verfügung stehen. Infolge zu geringer Fallzahlen kann dieses Alterssegment nicht weiter differenziert werden; allerdings deuten die Daten darauf hin, dass die Erwerbstätigenquote von Physikern bis kurz vor dem Erreichen der Altersgrenze von 65 Jahren noch bei ca. $70 \%$ liegt und erst mit dem Erreichen der Regelaltersgrenze der gesetzlichen Rentenversicherung - wie auch zu erwarten - stark sinkt. Doch selbst jenseits dieser Altersgrenze geht noch etwa jeder achte Physiker einer Erwerbstätigkeit nach. Häufig sind dies Personen, die in Teilzeit arbeiten, oder Selbstständige.

Aus den Erwerbstätigenquoten in den einzelnen Alterskohorten lässt sich ein demografiebedingter Ersatzbedarf errechnen, der zeigt, wie viele Physikerstellen in Zukunft altersbedingt aus dem Erwerbsleben ausscheiden und somit zur Aufrechterhaltung des Personalbestands ersetzt werden müssen. Bis zum Jahr 2019 sind dies ca. 2220 Physiker pro Jahr. Diese Zahl steigt in der Zukunft kontinuierlich an, bis auf ca. 3200 Personen pro Jahr in den Jahren 2030 bis 2034 . Hier geht es allein darum, diejenigen Personen, die ausscheiden, zu ersetzen. Tatsächlich jedoch ist die Anzahl erwerbstätiger Physiker zwischen 2005 und 2014 um durchschnittlich 2,3\% jedes Jahr gestiegen. Der resultierende expansionsbedingte Bedarf liegt folglich bei zusätzlichen rund 2300 Personen pro Jahr. 


\section{Berufe und Branchen}

Aus Tab. 2 ist zu erkennen, dass lediglich rund ein Fünftel aller Physiker im Erwerbsberuf Physiker arbeiten. Dies liegt daran, dass Physiker in anderen Berufen gern gesehen sind, aber auch an der sehr engen Definition des Erwerbsberufs Physiker. Ersteres ist nicht weiter verwunderlich, lernen Physiker doch im Studium, mathematisch-analytische Denkmuster auf hohem Niveau anzuwenden und auch in der Praxis komplexe technische Probleme zu lösen. Diese Fähigkeiten sind nicht allein auf das Lösen fachlicher Probleme begrenzt, sondern es sind Querschnittsthemen, die in vielen Berufen und Branchen Wichtigkeit haben. Auf die Definition des Erwerbsberufs Physiker wurde bereits am Anfang des Kapitels eingegangen

Erstmals sind mehr Physiker in einem Lehrberuf (in der Regel als Quer- und Seiteneinsteiger in den Lehrerberuf an einer weiterführenden Schule oder als Professor an einer Hochschule) als im klassischen Erwerbsberuf Physiker tätig. Aber auch in technisch-naturwissenschaftlichen Berufsfeldern sowie in Managementtätigkeiten finden viele Physiker sehr gute Beschäftigungschancen. Aus dem Mikrozensus geht ebenfalls hervor, dass knapp

Tab. 2 Erwerbstätige Physiker nach Erwerbsberuf (KIdB 2010), lexikografisch gereiht (Expertenberufe/sonstige Berufe) [2]

\begin{tabular}{lll}
\hline Erwerbsberufsgruppen & \multicolumn{2}{l}{ Erwerbstätige Physiker } \\
\cline { 2 - 3 } & Anzahl & Prozent \\
\hline Lehrberufe & 27.500 & 25,0 \\
Erwerbsberuf Physiker & 24.500 & 22,2 \\
Ingenieurberufe & 13.900 & 12,6 \\
Sonstige MINT-Expertenberufe & 11.400 & 10,4 \\
Sonstige Expertenberufe & $8100^{\mathrm{a}}$ & 7,3 \\
Wirtschaftswissenschaftliche & $5700^{\mathrm{a}}$ & 5,1 \\
$\quad$ Expertenberufe & 19.000 & 17,3 \\
Sonstige Berufe & 110.200 & 100 \\
Gesamt & &
\end{tabular}

aEingeschränkte statistische Zuverlässigkeit infolge geringer Zellbesetzung in der Stichprobe 
$83 \%$ der Physiker in sog. Expertenberufen tätig sind, für die typischerweise ein Hochschulstudium notwendig ist. Dies heißt aber nicht, dass die restlichen $20 \%$ in Berufen arbeiten, die keine anspruchsvolle Tätigkeit bieten oder in diesen nicht gefordert werden. Es heißt lediglich, dass für diese Berufe kein Hochschulstudium vorgeschrieben ist. Darunter fallen z. B. Tätigkeiten wie Schriftsteller oder Bundeskanzler. Und dort sind Physikerinnen bzw. Physiker ja hin und wieder anzutreffen.

Auch in Bezug auf Branchen streut die Erwerbstätigkeit von Physikern überaus weit (Tab. 3). Rund ein Viertel ist in der Industrie tätig, die restlichen drei Viertel in Branchen des Dienstleistungssektors, allen voran im Bildungssektor. Wie auch bei den Erwerbsberufen zeichnen in erster Linie attraktive Beschäftigungsmöglichkeiten (im Sinne eines positiven Pull-Faktors) für die hohe Beschäftigung von Physikern auch außerhalb der physiknahen Forschung und Entwicklung verantwortlich.

\section{Frauen in der Physik}

Laut Mikrozensus 2014 waren 15.700 oder 14,2 \% aller erwerbstätigen Physiker weiblich. Tab. 4 zeigt, dass der Anteil seit 2005 deutlich gestiegen ist. Im Vergleich stieg die Zahl erwerbstätiger Frauen um $59 \%$ an, die Zahl der erwerbstätigen Männer hingegen lediglich um $18 \%$.

Verglichen mit dem Frauenanteil unter den erwerbstätigen Akademikern, der 2014 ca. $45 \%$ betrug, wird deutlich, dass Frauen in der Physik immer noch unterrepräsentiert sind. Es gibt aber Grund zur Hoffnung. In die Studiengänge der Physik schreiben sich seit einigen Jahren mehr Frauen ein und schließen ihr Studium auch ab. Seit 2010 sind z. B. rund $20 \%$ der neu Promovierten weiblich [4].

Betrachtet man als Zahlengrundlage die Mitglieder der DPG, sind ebenfalls Steigerungen des Frauenanteils 
Tab. 3 Erwerbstätige Physiker nach Branche (Klassifikation der Wirtschaftszweige 2008 [3]), lexikografisch gereiht (Primär- und Sekundärsektor/Dienstleistungssektor) [2]

\begin{tabular}{|c|c|c|}
\hline \multirow[t]{2}{*}{ Wirtschaftszweig(e) } & \multicolumn{2}{|c|}{ Erwerbstätige Physiker } \\
\hline & Anzahl & Prozent \\
\hline $\begin{array}{l}\text { Metallindustrie (z. B. } \\
\text { Maschinen- und Fahrzeugbau) }\end{array}$ & 13.400 & 12,1 \\
\hline Elektroindustrie & 9900 & 9,0 \\
\hline $\begin{array}{l}\text { Sonstige Branchen des Primär- } \\
\text { und Sekundärsektors }\end{array}$ & $6300^{a}$ & 5,7 \\
\hline Erziehung und Unterricht & 27.800 & 25,2 \\
\hline $\begin{array}{l}\text { Wissensintensive technische } \\
\text { Dienstleistungen }\end{array}$ & 17.500 & 15,9 \\
\hline $\begin{array}{l}\text { Sonstige Branchen des Tertiär- } \\
\text { sektors }\end{array}$ & 15.600 & 14,2 \\
\hline $\begin{array}{l}\text { Informations- und } \\
\text { Kommunikationsdienst- } \\
\text { leistungen }\end{array}$ & 12.300 & 11,2 \\
\hline $\begin{array}{l}\text { Sonstige wissensintensive } \\
\text { Dienstleistungen }\end{array}$ & $7400^{a}$ & 6,8 \\
\hline Gesamt & 110.200 & 100 \\
\hline
\end{tabular}

${ }^{a}$ Eingeschränkte statistische Zuverlässigkeit infolge geringer Zellbesetzung in der Stichprobe

Tab. 4 Erwerbstätige Physiker nach Geschlecht [2]

\begin{tabular}{lllll}
\hline & Jahr: 2014 & \multicolumn{3}{c}{ Jahr: 2005 } \\
\cline { 2 - 5 } & $\begin{array}{l}\text { Erwerbstätige } \\
\text { Physiker }\end{array}$ & Prozent & $\begin{array}{l}\text { Erwerbstätige } \\
\text { Physiker }\end{array}$ & Prozent \\
\hline Weiblich & 15.700 & 14,2 & 9900 & 11,0 \\
Männlich & 94.500 & 85,8 & 79.900 & 89,0 \\
Gesamt & 110.200 & 100 & 89.800 & 100 \\
\hline
\end{tabular}

zu beobachten. Natürlich sind die DPG-Mitglieder nur eine Untergruppe der Physiker, und es sind sogar Nichtphysiker Mitglied. Um Trends zu beobachten, sind die Zahlen aber sicherlich aussagekräftig. Beobachtet man den Frauenanteil über die Jahre, ist doch immerhin eine enorme Steigerung von ca. $2 \%$ im Jahr 1982 bis zu ca. $15 \%$ im Jahr 2017 zu erkennen (Abb. 1). In Abb. 2 ist der Frauenanteil in den Alterskohorten der DPG auf- 


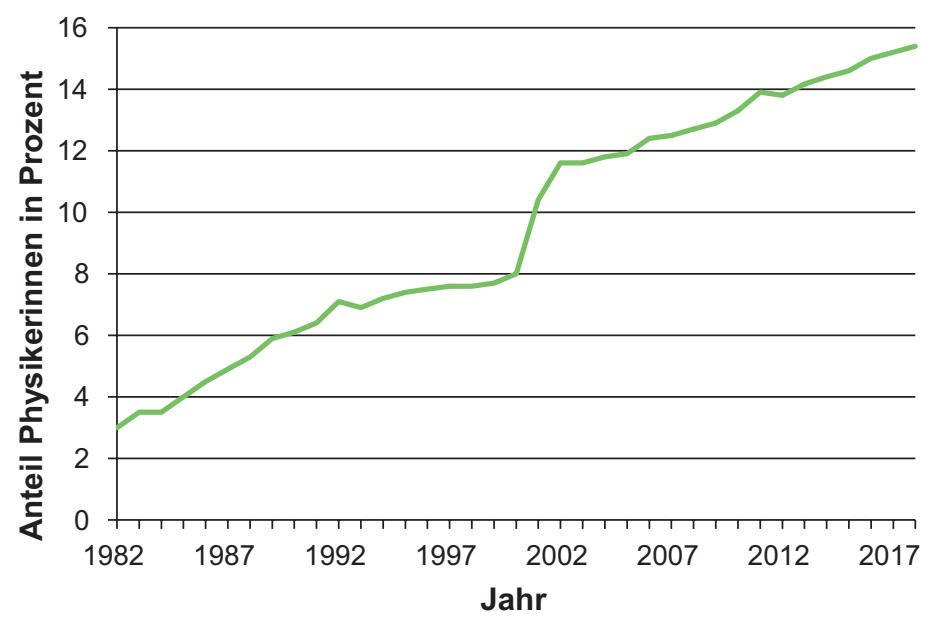

Abb. 1 Anteil von Physikerinnen in der Deutschen Physikalischen Gesellschaft (DPG) [5]

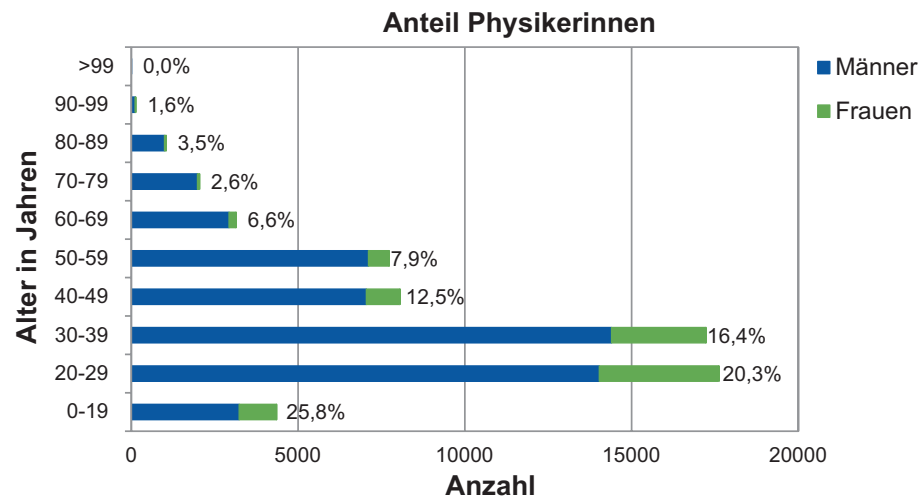

Abb. 2 Anteil von Physikerinnen in den verschiedenen Alterskohorten der Deutschen Physikalischen Gesellschaft (DPG) [5]

getragen. Dort ist $\mathrm{zu}$ sehen, dass unter den jüngeren DPG-Mitgliedern weitaus mehr Frauen sind als unter den älteren. Das macht Hoffnung, dass der Frauenanteil unter allen Physikern in Zukunft steigen wird. 


\section{Arbeitslosigkeit und Stellensuche}

Abb. 3 zeigt die Entwicklung der Arbeitslosenzahlen in der Physik vom Jahr 2000 bis zum Jahr 2017. Während die Zahl der Arbeitslosen bis zum Jahr 2007/2008 auf ein Minimum sank, stieg sie bis zum Jahr 2016 wieder an. In den letzten beiden Jahren hat sich dieser Trend umgekehrt. Von 2016 auf 2017 sank die Zahl der Arbeitslosen um $11 \%[6,7]$. Dieser Trend ist für Physiker selbstverständlich erfreulich, allerdings war die Situation auf dem Arbeitsmarkt auch in den Vorjahren nicht dramatisch, sondern im Vergleich zu manch anderer Berufsgruppe noch sehr komfortabel. Im beobachteten Zeitraum waren Physikerinnen und Physiker auf dem Arbeitsmarkt immer sehr gefragt. Die BA berechnet jährlich eine Arbeitslosenquote. Allerdings nicht ausschließlich für Physiker, sondern für physikalische und mathematische Erwerbsberufe gemeinsam. Im Jahr 2016 lag die Quote bei 2,9 \% [8]. Im gesamten

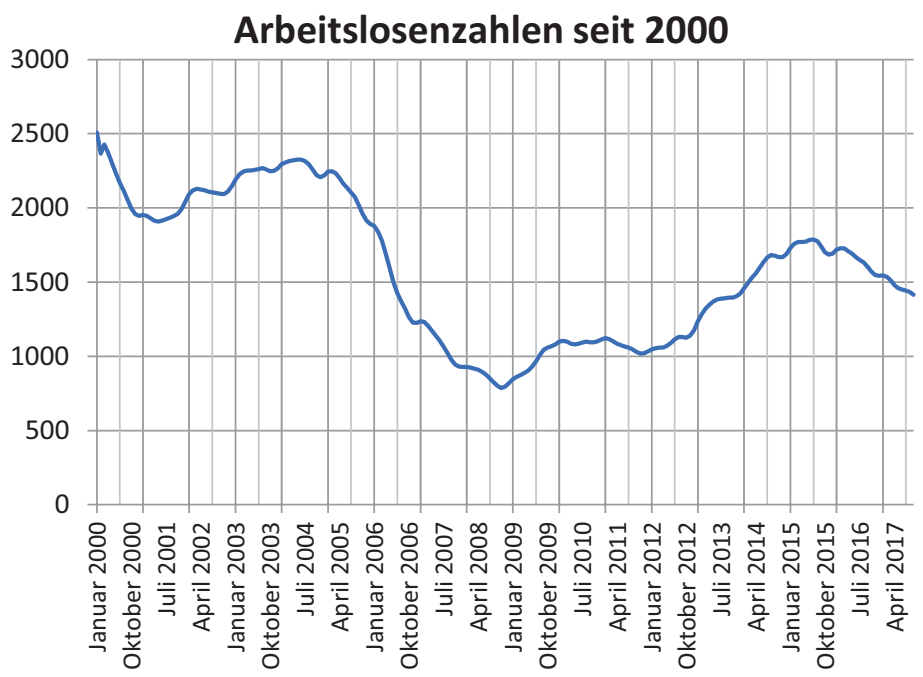

Abb. 3 Arbeitslose im Erwerbsberuf Physiker seit Januar 2000. Die Daten wurden über einen Zeitraum von fünf Monaten geglättet [6, 7] 
Akademikerbereich liegt sie zum Vergleich bei 2,6 \%, streut allerdings stark von 1,0\% (Human-und Zahnmedizin) bis 5,1\% (Biologie, Biochemie, Biotechnologie) [8].

Die bei der BA erhobenen Daten betreffen, wie oben bereits erläutert, diejenige Untergruppe von Physikern, die in die klassischen Physikberufe vermittelt werden möchte. Da diese Daten aber jährlich von der DPG analysiert werden können, eröffnen Sie die Möglichkeit, im Vergleich Trends festzustellen.

Bei der BA werden neben den Arbeitslosen auch gemeldete offene Stellen erfasst. Auch diese lassen sich von Jahr zu Jahr vergleichen. Bei der Interpretation ist allerdings zu bedenken, dass eine Analyse des Zukunftspanels des IW Köln aus dem Jahr 2010 für Mathematiker und Physiker einstellende Unternehmen ergeben hat, dass diese Unternehmen nur rund $10 \%$ der bei ihnen zu besetzenden Stellen dieser Berufsgruppe überhaupt an die BA melden. Grobe Trends im jährlichen Vergleich sind aber machbar.

In Abb. 4 werden die offenen Stellen in den größten Kategorien verglichen. Alle Zahlen sind auf das Jahr 2008 normiert. Hochschule sowie Forschung und Entwicklung $(\mathrm{F} \& \mathrm{E})$ in Industrie und Wirtschaft sind die Kategorien, in denen in allen Jahren am meisten offenen Stellen gemeldet werden. Das produzierende Gewerbe folgt mit leicht wechselnden Anteilen. Bis zum Jahr 2012 nahmen auch Personaldienstleistungen und Zeitarbeit einen großen Part ein; dieser Anteil hat sich in den letzten Jahren aber verringert.

\section{Physiker im Unternehmen}

Physikerinnen und Physiker arbeiten bevorzugt in größeren Unternehmen. Wie bereits im Jahr 2005 arbeiteten auch im Jahr 2014 nahezu drei Viertel in Unternehmen mit 50 oder mehr Mitarbeitern, das restliche Viertel in Unternehmen mit weniger als 50 Mitarbeitern (Tab. 5). In letzterer Unternehmenskategorie sind in der Regel auch jene 


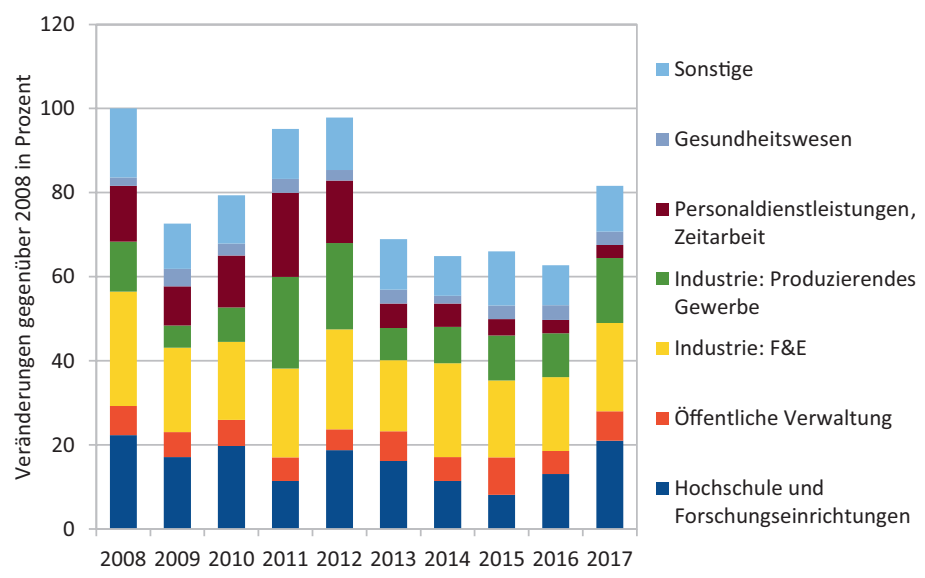

Abb. 4 Offene Stellen im Erwerbsberuf Physiker $[6,7]$

10 \% aller erwerbstätigen Physiker erfasst, die selbstständig sind. Abhängig beschäftigte Physiker sind folglich in der Regel bei mittleren bis größeren Unternehmen beschäftigt.

Mehr als ein Drittel aller erwerbstätigen Physiker sind in einer leitenden Position tätig, $17 \%$ als Führungskraft, d. h. mit Entscheidungsbefugnis über Personal, Budget und Strategie, weitere $17 \%$ als Aufsichtskraft, worunter das Anleiten und Beaufsichtigen von Personal, Verteilen und Kontrollieren von Arbeit verstanden wird.

\section{Berufszufriedenheit}

Einer der aussagefähigsten Indikatoren zur Beurteilung der Zufriedenheit im Beruf ist die rückblickende Bewertung der Studienfachwahl. Befragte man Physikerinnen und Physiker, gaben $87 \%$ an, dass sie wieder Physik studieren würden [7]. Damit weisen Physiker von allen Studiengängen - egal, ob Universität oder Fachhochschule die höchste Zufriedenheit mit ihrer Studienfachwahl auf. Im Durchschnitt aller Fachrichtungen würden nur $66 \%$ aller Absolventen das eigene Fach erneut studieren.

Also, Physik macht glücklich! 
Tab. 5 Erwerbstätige Physiker nach Unternehmensgröße ${ }^{a}$ [2]

\begin{tabular}{lllll}
\hline & Jahr: 2014 & \multicolumn{3}{c}{ Jahr: 2005 } \\
\cline { 2 - 5 } & Anzahl & Prozent & Anzahl & Prozent \\
\hline Bis 49 Mitarbeiter & 27.500 & 24,9 & 22.200 & 24,7 \\
50 bis 499 Mitarbeiter & 39.200 & 35,6 & 67.600 & 75,3 \\
$\begin{array}{l}500 \text { oder mehr Mit- } \\
\text { arbeiter }\end{array}$ & 43.600 & 39,5 & & \\
Gesamt & 110.200 & 100 & 89.800 & 100 \\
\hline
\end{tabular}

${ }^{\mathrm{a} A n z a h l}$ der tätigen Personen in dem Betrieb/der örtlichen Einheit

\section{Literatur}

1. Bundesagentur für Arbeit, 2011, Klassifikation der Berufe 2010 - Band 2: Definitorischer und beschreibender Teil, Nürnberg

2. Eigene Berechnungen auf Basis des Forschungsdatenzentrums (FDZ) der Statistischen Ämter des Bundes und der Länder, Mikrozensus 2014

3. 2010b, Klassifikation der Wirtschaftszweige (WZ 2008) des Statistischen Bundesamtes, 2010, Ausgabe 2008

4. G. Düchs und G. Ingold, Physik Journal, September 2018, S. 32

5. Mitgliedsdaten der Deutschen Physikalischen Gesellschaft e. V. Ähnliche und weitere Aufstellungen unter https:// www.dpg-physik.de/dpg/profil/struktur.html

6. U. Weigelt und A. Metzelthin, Physik Journal, Dezember 2017, S. 60

7. Bereitgestellte Daten der Bundesagentur für Arbeit

8. Statistik der Bundesagentur für Arbeit; Berichte: Blickpunkt Arbeitsmarkt - Akademikerinnen und Akademiker, Nürnberg, Juli 2017; bit.ly/2Af8Mkd

9. Briedis, Kolja, 2007, Übergänge und Erfahrungen nach dem Hochschulabschluss - Ergebnisse der HIS-Absolventenbefragung des Jahrgangs 2005, HIS Forum Hochschule 13/2007, URL: http://www.his.de/pdf/pub_fh/ fh-200713.pdf [Stand: 2009-04-10] 


\section{Weiterführende Literatur}

10 O. Koppel, Physikerinnen und Physiker im Beruf Anschlussstudie für die Jahre 2005 bis 2013 - Eine Studie im Auftrag der DPG, Bad Honnef (2016); www.dpg-physik.de/veroeffentlichung/broschueren/studien/arbeitsmarktstudie_2016.pdf

11 O. Koppel, Physikerinnen und Physiker im Beruf - Arbeitsmarktentwicklung, Einsatzmöglichkeiten und Demographie Eine Studie im Auftrag der DPG, Bad Honnef (2010); www. dpg-physik. de/veroeffentlichung/broschueren/studien/arbeitsmarkt_2010.pdf

Open Access Dieses Kapitel wird unter der Creative Commons Namensnennung 4.0 International Lizenz (http:// creativecommons.org/licenses/by/4.0/deed.de) veröffentlicht, welche die Nutzung, Vervielfältigung, Bearbeitung, Verbreitung und Wiedergabe in jeglichem Medium und Format erlaubt, sofern Sie den/die ursprünglichen Autor(en) und die Quelle ordnungsgemäß nennen, einen Link zur Creative Commons Lizenz beifügen und angeben, ob Änderungen vorgenommen wurden.

Die in diesem Kapitel enthaltenen Bilder und sonstiges Drittmaterial unterliegen ebenfalls der genannten Creative Commons Lizenz, sofern sich aus der Abbildungslegende nichts anderes ergibt. Sofern das betreffende Material nicht unter der genannten Creative Commons Lizenz steht und die betreffende Handlung nicht nach gesetzlichen Vorschriften erlaubt ist, ist für die oben aufgeführten Weiterverwendungen des Materials die Einwilligung des jeweiligen Rechteinhabers einzuholen.

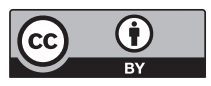

